Research Paper

\title{
Three-Step Hand-Assisted Laparoscopic D2 Radical Gastrectomy for Chinese Obese Patients: A Highly Efficient and Feasible Surgical Approach
}

\author{
JiaQing Gong ${ }^{* \bowtie}$, YongKuan $\mathrm{Cao}^{*}$, YongHua Wang, GuoHu Zhang, PeiHong Wang, GuoDe Luo \\ Center of General Surgery, the People's Liberation Army General Hospital of Chengdu Command, Chengdu 610083, Sichuan Province, \\ China. \\ *These authors have made equal contributions for this study. \\ $\square$ Corresponding author: JiaQing Gong, Center of General Surgery, the People's Liberation Army General Hospital of Chengdu Command, \\ Chengdu 610083, Sichuan Province, China. e-mail: cdgjq123 @126.com.
}

() Ivyspring International Publisher. This is an open-access article distributed under the terms of the Creative Commons License (http://creativecommons.org/ licenses/by-nc-nd/3.0/). Reproduction is permitted for personal, noncommercial use, provided that the article is in whole, unmodified, and properly cited.

Received: 2014.09.25; Accepted: 2014.11.15; Published: 2015.01.01

\begin{abstract}
Three-step hand-assisted laparoscopic D2 radical gastrectomy (HALG) is a modified surgical procedure that has achieved satisfactory results for obese patients in our surgical center. To fully elucidate the advantages of this procedure, in this study, comprehensive and in-depth comparative analyses were performed to assess clinical data from obese gastric cancer patients who underwent HALG, laparoscopic-assisted D2 radical gastrectomy (LAG), and open D2 radical gastrectomy $(O G)$ in our surgical center during a specific time period. For the 3 groups, incision length was 1.25 $\mathrm{cm}$ longer for the HALG group than for the LAG group but was significantly shorter for the HALG group than for the OG group $(P=0.00)$. The rate of conversion to laparotomy, the pneumoperitoneum time and the number of recovered lymph nodes were significantly better for the HALG group than for the LAG group $(P<0.05)$. The pain score at day 2 after surgery, intestinal function recovery time, and duration of postoperative hospital stay were not significantly different for the HALG and LAG groups $(P>0.05)$ but were significantly better for the HALG group than for the OG group $(P<0.05)$. There were significantly fewer postoperative complications for the HALG group than for the LAG and OG groups $(P=0.049)$. According to the results, the "three-step HALG method" incorporates both the thoroughness of the radical OG approach and the minimal invasiveness of the LAG approach for obese patients. Thus, the HALG approach is a relatively safe and extremely feasible surgical procedure for the treatment of these patients.
\end{abstract}

Key words: hand-assisted laparoscopic D2 radical gastrectomy, laparoscopy-assisted D2 radical gastrectomy, open D2 radical gastrectomy, obese patients.

\section{Introduction}

Obese gastric cancer patients exhibit the large accumulation and abnormal distribution of abdominal fat; these characteristics seriously affect the exposure of the operative field. In addition, perivascular fat parcels and lipid deposition on vascular walls lead to increased vascular fragility; as a result, slight stretching of blood vessels can easily lead to vascular rupture and bleeding, which severely affect the surgical process and increase surgical difficulty and risk. Furthermore, obesity is frequently associated with many cardiopulmonary and other chronic diseases that decrease operational tolerance [1]. Therefore, many scholars believe that caution is required with respect to the use of laparoscopic surgery for 
obese patients [2, 3]. However, laparoscopic-assisted D2 radical gastrectomy (LAG) has become a standard procedure for the radical treatment of advanced gastric cancer because of its unmatched advantages $[4,5]$. In recent years, many scholars have continued to investigate the use of LAG for obese gastric cancer patients and have obtained preliminary results $[6,7]$. However, the application of laparoscopic techniques among obese gastric cancer patients still faces the following bottlenecks. (1) It is difficult to expose the operative field, the control of bleeding is challenging, and operation times are lengthy [8]. (2) Laparoscopic surgeries are extremely difficult and risky, with a tortuous learning curve $[9,10]$; these issues severely affect the enthusiasm of surgeons for performing these operations.

To overcome the aforementioned technical bottlenecks, we have drawn upon the advantages of radical hand-assisted laparoscopic surgery for colon cancer [11] to perform hand-assisted laparoscopic D2 radical gastrectomy (HALG) and have developed the unique "three-step HALG method". To more thoroughly elucidate the advantages of the "three-step HALG method", in this study, systematic retrospective analyses of clinical data from obese gastric cancer patients who received HALG, LAG, and open D2 radical gastrectomy (OG) at our center during a particular time period were conducted, and the relevant findings were summarized.

\section{Material and methods}

General information D2 radical gastrectomy was performed in 270 cases involving obese gastric cancer patients at our center between July 2008 and December 2013. HALG, LAG, and OG were performed in 54, 51, and 165 of these cases, respectively. According to the standards established by the World Health Organization for Asian obese patients in 2003[12] and the surgical treatment guidelines for Chinese obese patients in 2007[13], obesity is defined as a body mass index $(\mathrm{BMI}) \geq 25 \mathrm{~kg} / \mathrm{m}^{2}$. A study design of a non-randomized controlled trial during the examined time period was adopted for this investigation, with surgical methods chosen based on the condition and wishes of each patient. The following case inclusion criteria were utilized: (1) there was a definite preoperative pathological diagnosis; (2) distal metastasis was excluded by preoperative auxiliary examinations, the tumor had not directly invaded adjacent organs, and complete resection of the tumor appeared to be feasible; and (3) R0 resection was confirmed by postoperative pathological examination. Cases were excluded if intraoperative findings revealed peritoneal dissemination of the tumor or if D2 radical surgery could not be definitively completed. General information includes patients' ages, genders, BMI values, American Society of Anesthesiologists (ASA) physical status classifications[14], major associated underlying preoperative diseases (hypertension, pulmonary insufficiency, or diabetes mellitus), history of abdominal surgery, tumor sizes, tumor-node-metastasis (TNM) stages, and rates of conversion to laparotomy. All surgeries were completed by highly qualified specialists who possessed extensive clinical experience. The same postoperative treatment principles and discharge criteria were applied for all patients.

Surgical methods Tumor stages were determined based on abdominal exploration. The dissection of lymph nodes in the perigastric region and D2 radical gastrectomy were performed in accordance with the Japanese publication "Gastric cancer treatment protocols". Conventional OG and LAG were mature and well-established surgical procedures (Fig.1 E, F). The "three-step HALG method" was divided into three steps (Fig.1 A D): hand-assisted incision surgeries under direct vision, hand-assisted laparoscopic surgeries, and digestive tract reconstruction. The specific surgical procedures and our preliminary work have been published in Surgical Endoscopy [15].

Observation indicators Full-time personnel at our center completed the observation and recording of all indicators. Intraoperative indicators included type of surgery (total gastrectomy, proximal gastrectomy, or distal gastrectomy), operation time (total operation time and laparoscopic operation time), quantity of blood lost, incision length, number of lymph nodes recovered, and unexpected intraoperative injury. Postoperative indicators included postoperative pain scores (at days 1 and 2 after surgery), intestinal function recovery time, duration of postoperative hospital stay, complications (pulmonary infection, arrhythmia, venous thrombosis, gastrointestinal fistula, disorders of gastrointestinal function, bile reflux, abdominal cavity infections, and wound infections), reoperation rate, readmission rate within 30 days after surgery, and mortality rate. A pathologist and a surgeon removed lymph nodes from resected specimens individually, which were classified by pathological examination. Postoperative pain scores were assessed using the visual analog scale (VAS) [16]. Pain scores were evaluated three times per day, and the highest score for a day was used as the day's pain score. Cases involving conversion to laparotomy were not included in intraoperative and postoperative statistics.

\section{Statistical analysis}

The SPSS16.0 software package was used for 

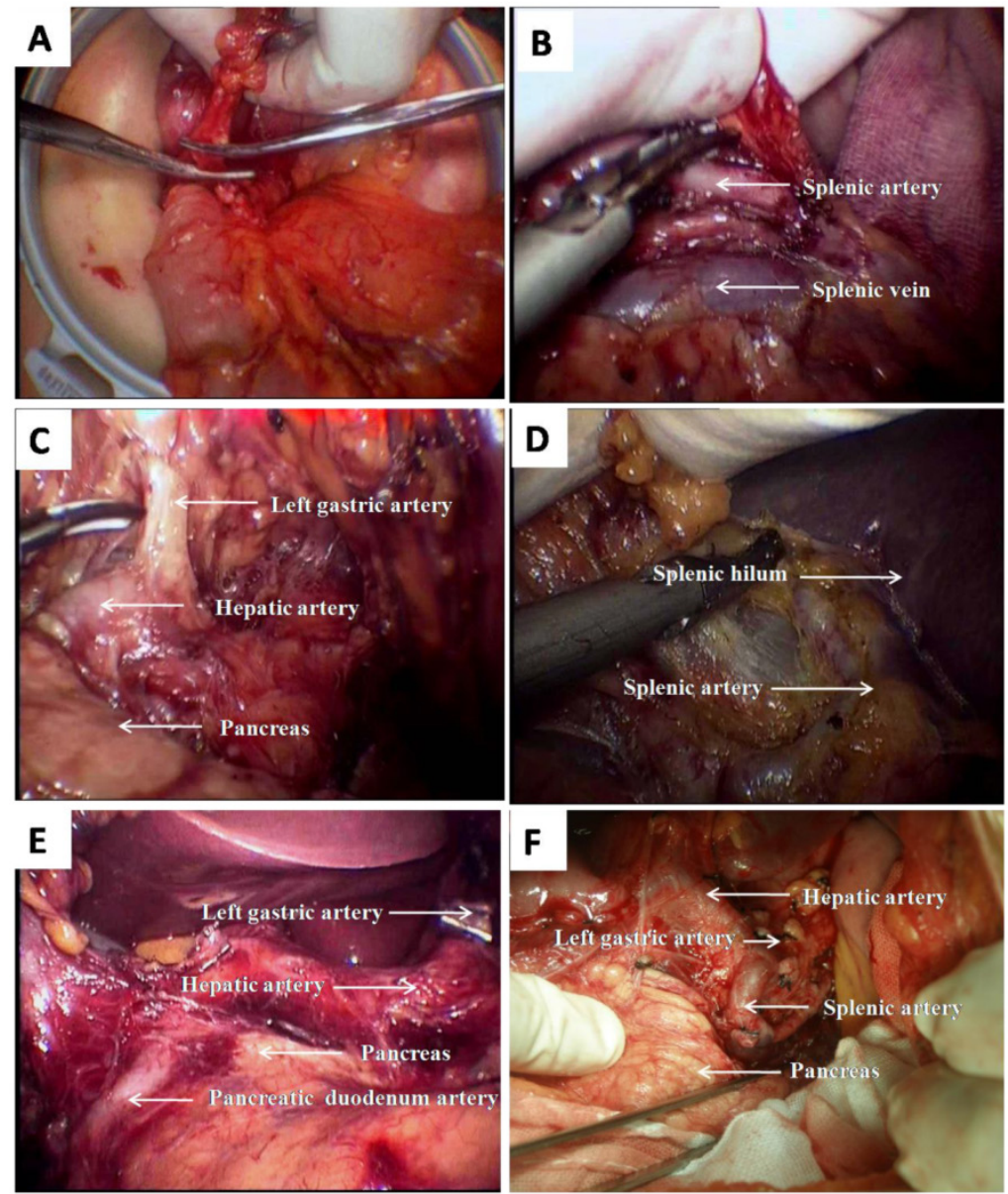

Figure 1. HALG for total gastrectomy (A D): A shows that the NO. 6 lymph nodes were dissected under direct vision via auxiliary incision, and right gastroepiploic artery was transected; B shows that the NO. 11 lymph nodes were dissected, and Splenic vessels was revealed; $C$ shows that the NO. 7,8 and 9 lymph nodes were dissected, and Left gastric artery and hepatic artery were revealed; D shows that NO.10 lymph nodes were dissected, and splenic hilum and splenic artery were revealed. LAG for antral cancer (E): $E$ shows that the NO. 5,7,8,9 and 12 lymph nodes were dissected, and Left gastric artery, hepatic artery and pancreatic duodenum artery were revealed. OG for antral cancer $(F)$ : $F$ shows that the NO. 5,7,8,9,11 and 12 lymph nodes were dissected, and Left gastric artery, hepatic artery and splenic artery were revealed.

statistical analyses. Measurement data were expressed as meanststandard deviations and were analyzed by one-way analysis of variance (ANOVA), and pairwise comparisons between groups were performed with LSD method. Count data were assessed using $X^{2}$ tests. $P<0.05$ was adopted as the threshold for significance in statistical analyses.

\section{Results}

\section{General information results}

In this study, there were 54, 51, and 165 cases in the HALG, LAG, and OG groups, respectively. The results of statistical analyses for patients' general information are presented in Table 1 . In particular, the $P$ values for age, gender, BMI, and ASA were 0.21, 0.16, 0.08 , and 0.70 , respectively. The major underlying diseases included hypertension, pulmonary insufficiency, and diabetes mellitus; the $P$ values for these diseases were $0.57,0.45$, and 0.57 , respectively. The $P$ values for a history of pulmonary surgery, tumor size, and the TNM stages were $0.22,0.13$, and 0.27 , respectively. Conversion to laparotomy occurred in 1 case in the HALG group and 8 cases in the LAG group, and $P=0.03$. Conversion to laparotomy occurred for the following reasons: 1 case in the HALG group of massive bleeding from an injury in the left gastric artery near the splenic hilum, 5 cases in the LAG group of intraoperative bleeding, 2 cases in the LAG group of tumor invasion of the roots of the middle colic artery, and 1 case in the LAG group of invasion of the duodenum by a tumor in the gastric antrum. None of the cases involving conversion to laparotomy were included in the intraoperative and postoperative statistics; therefore, 53 cases in the HALG group and 43 cases in the LAG group were included in the intraoperative and postoperative statistical analyses.

\section{Results for intraoperative indicators}

A total of 6 intraoperative indicators were examined, and the results of statistical analyses are presented in Table 2. $P$ $=0.00$ for comparisons of incision length among the groups. In particular, the incision length for the HALG group was $7.04 \pm 0.19 \mathrm{~cm}$, which was $1.25 \mathrm{~cm}$ longer than that for the LAG group and $6.82 \mathrm{~cm}$ shorter than that for the OG group. $P=0.73$ for the comparison of type of surgery among the experimental groups. Total operation time for the HALG group was $179.11 \pm 16.82 \mathrm{~min}$, which was $25 \mathrm{~min}$ shorter than that for the LAG group $(P=0.00)$ and 8 min longer than that for the OG group $(P=0.76)$. Laparoscopic operation time for the HALG group was $50.92 \pm 9.89$ min; which was 119 min shorter than that for the LAG group $(P=0.00)$. $P=0.77$ for the comparison of intraoperative blood loss among the experimental groups. The number of lymph nodes recovered for the HALG group was 19.36 \pm 3.27 , which was 3 more than that for the LAG group $(P=0.00)$ and 1 more than that for the OG group $(P=0.21) . P=0.09$ for the comparison of unexpected injuries among the experimental groups. In particular, there were 2 cases of such injuries in the HALG group (with unexpected injuries occurring in $3.77 \%$ of the cases in this group), including 1 case of mesocolon injury and 1 case of minor lacerations to the lower pole of the spleen. There were 6 cases of unexpected injuries in the OG group, with 
unexpected injuries occurring in $3.64 \%$ of the cases in this group, and 5 cases of unexpected injuries in the LAG group, with unexpected injuries occurring in $11.63 \%$ of the cases in this group.

\section{Postoperative indicator results}

A total of 7 postoperative indicators were examined, and the results of statistical analyses are presented in Table 3. The VAS score for the HALG group on day 1 after surgery was $5.46 \pm 1.43$, which was significantly higher than that for the LAG group $(P=0.00)$, but significantly lower than that for the OG group ( $P=0.02)$. The VAS score for the HALG group on day 2 after surgery was $4.14 \pm 1.12$, which was not significantly different from that for the LAG group $(P$ $=0.13$ ) but was significantly lower than that for the OG group ( $P=0.00$ ). The postoperative hospital stay duration for the HALG group was $9.64 \pm 2.34$ days, which was similar to that for the LAG group $(P=0.98)$ but was 1 day less than that for the OG group $(P=0.01)$. The intestinal function recovery time for the HALG group was $62.81 \pm 14.61 \mathrm{~h}$, which was not significantly different from that for the LAG group $(P=0.80)$ but was $47 \mathrm{~h}$ less than that for the OG group $(P=0.00)$.

Table 1. The comparison of general data.

\begin{tabular}{|c|c|c|c|c|}
\hline & HALG(n=54) & LAG(n=51) & $O G(n=165)$ & $P$ Value \\
\hline Age(y),mean \pm SD & $56.09 \pm 9.24$ & $54.02 \pm 8.10$ & $53.07 \pm 11.95$ & 0.21 \\
\hline Median, Range & $55.5(42-72)$ & $54(39-67)$ & $53(34-76)$ & \\
\hline Sex ratio(male to female) & $23: 31$ & $24: 27$ & $93: 72$ & 0.16 \\
\hline BMI, mean \pm SD & $29.71 \pm 2.44$ & $28.67 \pm 2.33$ & $29.87 \pm 3.80$ & 0.08 \\
\hline Median, Range & $30.00(25.2-33.6)$ & $28.2(25.1-32.9)$ & $28.60(25.1-42.9)$ & \\
\hline ASA & & & & 0.7 \\
\hline I & 6 & 5 & 21 & \\
\hline II & 35 & 37 & 118 & \\
\hline III & 13 & 9 & 26 & \\
\hline \multicolumn{5}{|l|}{ The main underlying diseases } \\
\hline Hypertension & 9 & 9 & 37 & 0.57 \\
\hline Pulmonary insufficiency & 10 & 6 & 32 & 0.45 \\
\hline Diabetes & 4 & 7 & 17 & 0.57 \\
\hline Previous abdominal operation, $\mathbf{n}(\%)$ & $4(7.41 \%)$ & $3(5.88 \%)$ & $22(13.33 \%)$ & 0.22 \\
\hline Size of tumor $(\mathrm{cm})$, mean \pm SD & $4.69 \pm 1.36$ & $4.14 \pm 1.39$ & $4.43 \pm 1.41$ & 0.13 \\
\hline Median, Range & $4.82(2.0-6.9)$ & $4.20(1.7-7.0)$ & $4.40(1.10-7.00)$ & \\
\hline TNM stage (n) & & & & 0.27 \\
\hline I & 8 & 5 & 25 & \\
\hline II & 10 & 18 & 30 & \\
\hline IIIA & 12 & 12 & 41 & \\
\hline IIIB & 11 & 11 & 33 & \\
\hline IV & 13 & 5 & 36 & \\
\hline Open conversion (n \%) & $1(1.85 \%)$ & $8(15.69 \%)$ & & 0.03 \\
\hline
\end{tabular}

ASA: American Society of Anesthesiologists, BMI: body mass index (calculated as kg/m²), TNM: tumor-node-metastasis, HALG: hand-assisted laparoscopic D2 radical gastrectomy.

Table 2. The comparison of intraoperative data.

\begin{tabular}{|c|c|c|c|c|c|c|}
\hline \multirow[t]{2}{*}{ Cohort } & \multirow[t]{2}{*}{$\operatorname{HALG}(n=53)$} & \multirow[t]{2}{*}{ LAG $(n=43)$} & \multirow[t]{2}{*}{$\mathrm{OG}(\mathrm{n}=165)$} & \multicolumn{3}{|c|}{$P$ Value } \\
\hline & & & & $P$ & $P 1$ & $P 2$ \\
\hline \multicolumn{7}{|l|}{ Incision length(cm) } \\
\hline mean $\pm S D$ & $7.04 \pm 0.19$ & $5.83 \pm 0.60$ & $13.80 \pm 0.90$ & 0 & 0 & 0 \\
\hline Median, Range & $7.09(6.7-7.3)$ & $5.84(4.8-6.8)$ & 13.91(12.3-15.4) & & & \\
\hline Type of operation(n) & & & & 0.73 & 0.81 & 0.31 \\
\hline Total gastrectomy & 16 & 13 & 55 & & & \\
\hline Distal gastrectomy & 30 & 25 & 81 & & & \\
\hline Proximal gastrectomy & 7 & 5 & 29 & & & \\
\hline \multicolumn{7}{|l|}{ Operative time(min) } \\
\hline mean $\pm S D($ Total $)$ & $179.11 \pm 16.82$ & $207.21 \pm 18.69$ & $171.84 \pm 9.41$ & 0 & 0 & 0.76 \\
\hline Median, Range & 181(147 209) & 206(180 237) & $173(155 \sim 186)$ & & & \\
\hline mean $\pm S D($ Lap $)$ & $50.92 \pm 9.89$ & $172.23 \pm 16.78$ & & 0 & & \\
\hline Median, Range & $51(35 \sim 68)$ & $170(146 \sim 204)$ & & & & \\
\hline \multicolumn{7}{|l|}{ Blood loss(mL) } \\
\hline mean $\pm S D$ & $249.06 \pm 68.87$ & $257.51 \pm 76.20$ & $256.91 \pm 72.95$ & 0.77 & 0.57 & 0.49 \\
\hline Median, Range & $245(126 \sim 364)$ & $268(129 \sim 363)$ & $259(122 \sim 378)$ & & & \\
\hline \multicolumn{7}{|c|}{ Lymph nodes harvested } \\
\hline mean $\pm S D$ & $19.36 \pm 3.27$ & $16.37 \pm 3.73$ & $18.68 \pm 3.46$ & 0 & 0 & 0.21 \\
\hline Median, Range & $20(14 \sim 25)$ & $17(10 \sim 22)$ & $19(13 \sim 24)$ & & & \\
\hline Unexpected-injury & $2(3.77 \%)$ & $5(11.63 \%)$ & $6(3.64 \%)$ & 0.09 & 0.14 & 0.96 \\
\hline
\end{tabular}

HALG: hand-assisted laparoscopic D2 radical gastrectomy; P1: HALG vs. LAG; P2: HALG vs. OG. 
Table 3. The comparison of postoperative data.

\begin{tabular}{|c|c|c|c|c|c|c|}
\hline \multirow[t]{2}{*}{ Cohort } & \multirow[t]{2}{*}{ HALG(n=53) } & \multirow[t]{2}{*}{$\mathrm{LAG}(\mathrm{n}=43)$} & \multirow[t]{2}{*}{$\mathrm{OG}(\mathrm{n}=165)$} & \multicolumn{3}{|c|}{$P$ Value } \\
\hline & & & & $P$ & $P 1$ & $P 2$ \\
\hline \multicolumn{7}{|l|}{ VAS, mean $\pm S D$} \\
\hline D1 & $5.46 \pm 1.43$ & $4.24 \pm 1.51$ & $6.34 \pm 2.49$ & 0 & 0 & 0.02 \\
\hline D2 & $4.14 \pm 1.12$ & $3.82 \pm 0.85$ & $5.43 \pm 1.77$ & 0 & 0.13 & 0 \\
\hline Length of stay(d), mean $\pm S D$ & $9.64 \pm 2.34$ & $9.65 \pm 1.80$ & $10.56 \pm 2.18$ & 0 & 0.98 & 0.01 \\
\hline Median, Range & $9(7-17)$ & $9(7-13)$ & $10(8-18)$ & & & \\
\hline \multicolumn{7}{|l|}{ functional recovered of bowel (h) } \\
\hline mean $\pm S D$ & $62.81 \pm 14.61$ & $63.65 \pm 17.41$ & $109.66 \pm 28.26$ & 0 & 0.8 & 0 \\
\hline Median, Range & 61(39-98) & $59(38-100)$ & $108(65-157)$ & & & \\
\hline Complications & $5(9.43 \%)$ & $11(25.58 \%)$ & $41(24.85 \%)$ & 0.049 & 0.07 & 0.03 \\
\hline Pulmonary infection & $2(3.77 \%)$ & $4(9.30 \%)$ & $6(3.64 \%)$ & 0.27 & & \\
\hline arrhythmia & $1(1.89 \%)$ & $3(6.98 \%)$ & $5(3.03 \%)$ & 0.35 & & \\
\hline Venous thrombosis & 0 & 0 & $2(1.21 \%)$ & 0.56 & & \\
\hline Anastomotic leak(n \%) & $1(1.89 \%)$ & $2(4.65 \%)$ & $5(3.03 \%)$ & 0.74 & & \\
\hline Gastrointestinal dysfunction & $1(1.89 \%)$ & $2(4.65 \%)$ & $8(4.85 \%)$ & 0.64 & & \\
\hline Bile back flow & 0 & 0 & $5(3.03 \%)$ & 0.23 & & \\
\hline Abdominal cavity infection(n \%) & 0 & 0 & $4(2.42 \%)$ & 0.31 & & \\
\hline Wound infect(n \%) & 0 & 0 & $6(3.64 \%)$ & 0.17 & & \\
\hline Reoperation(n \%) & $1(1.89 \%)$ & $1(2.33 \%)$ & $2(1.21 \%)$ & 0.85 & & \\
\hline Readmission & $2(3.77 \%)$ & $2(4.65 \%)$ & $8(4.85 \%)$ & 0.95 & & \\
\hline Mortality & 0 & 0 & $2(1.21 \%)$ & 0.56 & & \\
\hline
\end{tabular}

HALG: hand-assisted laparoscopic D2 radical gastrectomy; P1: HALG vs. LAG; P2: HALG vs. OG.

For each individual complication, $P>0.05$ for comparisons among the experimental groups. The HALG group included a total of 5 cases of postoperative complications, with postoperative complications occurring in $9.43 \%$ of the cases in this group; this percentage was significantly lower than the corresponding percentages of $25.58 \%$ for the LAG group and $24.85 \%$ for the OG group $(P=0.049)$. In particular, $P=0.07$ for the comparison of postoperative complications between the HALG and LAG groups, and $P$ $=0.03$ for the comparison of postoperative complications between the HALG and OG groups. There were 3 cases of cardiopulmonary complications in the HALG group, and such complications occurred in $5.66 \%$ of the cases in this group. In the LAG group, cardiopulmonary complications occurred in $16.28 \%$ of cases, and $P=0.00$ for the comparison of the rates of cardiopulmonary complications between the HALG and LAG groups. In the OG group, cardiopulmonary complications occurred in $6.67 \%$ of cases. Postoperative gastrointestinal fistula is a severe complication $(P$ $=0.74)$ that occurred in 1 case $(1.89 \%)$ in the HALG group, 2 cases $(4.65 \%)$ in the LAG group, and 5 cases $(3.03 \%)$ in the OG group. There were 1, 1, and 2 cases of reoperation for peritoneal drainage in the HALG, LAG, and OG groups, respectively. $P=0.85$ for the comparison of reoperation rates among all groups. B-mode ultrasound was used to guide the placement of drainage tubes in patients who were not subjected to second surgeries.

In the HALG, LAG, and OG groups, there were 2,2 , and 8 cases, respectively, of patients who were readmitted within 30 days of the surgery; such cases accounted for $3.77 \%, 4.65 \%$, and $4.85 \%$ of the cases in each respective group $(P=0.95)$. In the HALG group, 1 patient was readmitted due to intestinal adhesion, and 1 patient was readmitted due to abdominal distention after eating. After these 2 patients received 2-5 days of treatment, all of their symptoms had been relieved, and the patients were discharged. There were no postoperative deaths in either the HALG or the LAG groups, whereas there were 2 cases of postoperative death in the OG group (with such cases accounting for $1.21 \%$ of this group). One patient died due to the failure of cardiopulmonary function, and the other patient, who was in poor general condition, died of abdominal infection caused by poor drainage from a gastrointestinal fistula. $P=0.56$ for a comparison of postoperative deaths among the experimental groups.

\section{Discussion}

Laparoscopic techniques have produced positive outcomes for the radical treatment of obese gastric cancer patients, however, the following shortcomings continue to limit the use of laparoscopic approach: (1) laparoscopic approaches involve prolonged operation times, resulting in fatigue among surgeons and a tortuous learning curve for these techniques[8-10]; (2) the effects of radical laparoscopic surgery have been questioned[17]; and (3) prolonged pneumoperitoneum aggravates cardiopulmonary burdens, leading to increased perioperative cardiopulmonary complications $[18,19]$. To realize the advantages but avoid the disadvantages of laparotomies and laparoscopies, we developed the unique "three-step HALG method". 
Through systematic retrospective analyses and summarization of the clinical data from all patients of this study, we believe that the "three-step HALG method" has unique advantages for obese gastric cancer patients and is an extremely viable option for the surgical treatment of these patients.

\section{The unique advantages of auxiliary incision for the "three-step HALG method"}

The laparoscopic procedures of LAG surgery can be unusually difficult in cases involving obese patients because the greater omentum occupies a large volume of space in the upper abdomen [8]. However, HALG begins by creating an incision below the xiphoid process. This incision not only is used for hand-assisted laparoscopic operations, tissue remov$\mathrm{al}$, and digestive tract reconstruction but also provides the unique advantage of enabling partial tissue separation and perigastric lymph node dissection to be completed under direct vision. The incision created using a hand-assisted device with a blue butterfly base can fully expose the local operative field. First, the transverse colon and the greater omentum are lifted outside the incision; the greater omentum is then fully freed and removed and this removal allows for the fuller and clearer exposure of the operative field under direct vision. The surgery continues by freeing the anterior lobe of the transverse mesocolon, part of the pancreatic capsule, and the duodenal bulb. Dissection is then performed on the lymph nodes in groups 5, 6, 12a, and $14 \mathrm{v}$ and a portion of the lymph nodes in group 8a.This approach greatly reduce pneumoperitoneum and laparoscopic operation time.

When completed the first step, the upper abdominal space is largely released and surgeons can more freely conduct left hand-assisted operations in the second step of the surgery. Surgeon can use the sensitive direct touch of the left hand and can achieve separation with the assistance of ultrasonic scalpels; as a result, the separation of major blood vessels and lymph node dissection can be more easily accomplished. During LAG surgery, the dissection of the lymph nodes in groups $7,8,9,10$, and 11p presents operational difficulties[20-22]. During the HALG surgery, the assisting hand fully exposes the operative field, and the thumb and index finger can directly touch and protect major blood vessels and tissues wrapped in fat tissue, providing important advantages in the dissection of the region from the lesser curvature of the stomach and the splenic artery to the splenic hilum and the gastric cardia. Therefore, there is significantly less difficulty and risk involved in the surgical procedure for HALG than for LAG.

\section{The thoroughness of radical treatment for the "three-step HALG method"}

The number of dissected perigastric lymph nodes is an important criterion for measuring the thoroughness of radical treatment [23, 24]. In this study, the number of dissected lymph nodes was smaller than that in previous studies [25], which were possible associated with that the other authors used lymphatic tracer during surgery, or our way of harvested lymph nodes from specimen need to be improved. In this study, similar numbers of lymph nodes were recovered for both the HALG and OG groups $(P=0.21)$. The number of lymph nodes recovered for the LAG group was 3 nodes fewer than that for the HALG group $(P=0.00)$. Therefore, we believe that the HALG approach was more thorough than the LAG approach. The reasons maybe as follow: the lymph node groups 6, 7, 8a, 9, 10, and 11 are in close proximity to major blood vessels which are encapsulated by fat tissues, and severe unexpected injuries can readily occur during lymph node dissection; therefore, significant difficulties will accompany with LAG surgery [20-22]. In HALG surgery, dissection of lymph nodes in group 6 and a portion of 8 a can be conducted under direct vision. In addition, during the laparoscopic portion of the HALG surgery, the tactile, exposure, and protection roles of the hands can be completely fulfilled; therefore, the separation and cutting functions of ultrasonic scalpels can be fully realized, and the complexity of surgical procedures can be significantly reduced. As a result, relatively thorough lymph node dissection can be achieved.

\section{The minimal invasiveness for the "three-step HALG method"}

The question of whether hand-assisted laparoscopic surgery is minimally invasive with respect to damage to the body remains unresolved in the extant literature [26, 27]. We believe that HALG causes fewer traumas than OG and exhibits the same minimal invasiveness as LAG. (1) The incision length for the HALG group significantly shorter than that for the OG group $(P=0.00)$, and only $1.25 \mathrm{~cm}$ longer than that for the LAG group. (2) Pneumoperitoneum time for the HALG group was much lower than that for the LAG group $(P=0.00)$. Because of this prolonged pneumoperitoneum time, there were significant compression effects on the hearts and lungs of obese patients in the LAG group, and increased postoperative cardiopulmonary complications were a direct result of this issue $[18,19]$. (3) The VAS scores for the HALG group on day 2 after surgery did not differ from that for the LAG group $(P=0.13)$, but remained significantly lower than that for the OG group ( $P$ $=0.00)$. These findings indicated that similar levels of 
pain control were achieved for HALG and LAG. (4) Intestinal function recovery time and duration of postoperative hospital stay did not significantly differ for the HALG and LAG groups $(P>0.05)$ but were significantly shorter for the HALG group than for the OG group $(P<0.05)$. These results suggested that with respect to abdominal organs, HALG and LAG were significantly less intrusive than OG.

\section{The safety for the "three-step HALG method"}

The disturbance, traction and the compression of the thoracic cavity by pneumoperitoneum during surgery are the important elements of trauma [28]. Studies have demonstrated that trauma is closely associated with the stability of the internal environment $[29,30]$. Obese patients suffer from many underlying diseases and exhibit reduced cardiopulmonary function and operational tolerance; thus, their internal environment is relatively sensitive to trauma [31]. In this study, HALG significantly shorten pneumoperitoneum time. Therefore, HALG is a relatively safe surgical approach. (1) The rates of unexpected intraoperative injuries in the HALG and OG groups were markedly lower than that in the LAG group. (2) The rate of complications in the HALG group was significantly lower than that in the LAG and OG groups $(P=0.049)$. In-depth analyses revealed that the percentage of cases involving postoperative cardiopulmonary complications was significantly lower for the HALG group than that for the LAG group ( $P$ $=0.00$ ). (3) There was one case of conversion to laparotomy in the HALG group; which was markedly lower than the corresponding percentage for the LAG group $(P=0.03)$. These results indicated that the assisting hand in the HALG surgery played important roles in protecting, dissecting, and separating critical fat-encapsulated structures, resulting in better control of surgery-related injury. (4) There were no significant differences among the experimental groups with respect to quantity of blood lost, reoperation rate, or readmission rate within 30 days after surgery $(P$ $>0.05$ ).

\section{Abbreviations}

HALG: hand-assisted laparoscopic D2 radical gastrectomy

LAG: laparoscopic-assisted D2 radical gastrectomy

OG: open D2 radical gastrectomy

BMI: obesity is defined as a body mass index

ASA: American Society of Anesthesiologists

TNM: tumor-node-metastasis

\section{Competing Interests}

The authors have declared that no competing interest exists.

\section{References}

1. Harris AT, Morell D, Bajaj Y, et al. A discussion of airway and respiratory complications along with general considerations in obese patients. Int J Clin Pract. 2010;64:802-6.

2. Kunisaki C, Makino $H$, Takagawa $R$, et al. Predictive factors for surgical complications of laparoscopy-assisted distal gastrectomy for gastric cancer. Surg Endosc. 2009;23:2085-93.

3. Kim MG, Kim KC, Kim BS, et al. A totally laparoscopic distal gastrectomy can be an effective way of performing laparoscopic gastrectomy in obese patients (body mass index $\geq 30$ ). World J Surg. 2011;35:1327-32.

4. Lee MS, Lee JH, Park do J, et al. Comparison of short- and long-term outcomes of laparoscopic-assisted total gastrectomy and open total gastrectomy in gastric cancer patients. Surg Endosc. 2013;27:2598-605.

5. Meng H, Zhou D, Jiang X, et al. Incidence and risk factors for postsurgical gastroparesis syndrome after laparoscopic and open radical gastrectomy. World J Surg Oncol. 2013 ;11:144.

6. Sugimoto M, Kinoshita T, Shibasaki H, et al. Short-term outcome of total laparoscopic distal gastrectomy for overweight and obese patients with gastric cancer. Surg Endosc. 2013;27:4291-6.

7. Kim MG, Kawada H, Kim BS, et al. A totally laparoscopic distal gastrectomy with gastroduodenostomy (TLDG) for improvement of the early surgical outcomes in high BMI patients. Surg Endosc. 2011;25:1076-82.

8. Miyaki A, Imamura K, Kobayashi R, et al. Impact of visceral fat on laparoscopy-assisted distal gastrectomy. Surgeon. 2013;11:76-81.

9. Yoshikawa K, Shimada M, Kurita N, et al. Visceral fat area is superior to body mass index as a predictive factor for risk with laparoscopy-assisted gastrectomy for gastric cancer. Surg Endosc. 2011;25:3825-30.

10. Zhou D, Quan Z, Wang J, et al.Laparoscopic-assisted versus open distal gastrectomy with $\mathrm{D} 2$ lymph node resection for advanced gastric cancer: effect of learning curve on short-term outcomes. a meta-analysis. J Laparoendosc Adv Surg Tech A. 2014;24:139-50.

11. Meshikhes AW. Controversy of hand-assisted laparoscopic colorectal surgery. World J Gastroenterol. 2010;16:5662-8.

12. Choo V. WHO reassesses appropriate body-mass index for Asian populations. Lancet. 2003;361:85.

13. Zheng CZ. The surgical treatment guidelines for Chinese obese patients. Chin J Pract Surg. 2007;27:759-62.

14. Tan WP, Talbott VA, Leong QQ, et al. American Society of Anesthesiologists class and Charlson's Comorbidity index as predictors of postoperative colorectal anastomotic leak: a single-institution experience. J Surg Res. 2013;184:115-9.

15. Gong JQ, Cao YK, Li YM, et al. Hand-assisted laparoscopic vs. laparoscopy-assisted D2 radical gastrectomy: a prospective study. Surg Endosc. 2014; 28:2998-3006.

16. Aicher B, Peil H, Peil B, et al. Pain measurement: Visual Analogue Scale (VAS) and Verbal Rating Scale (VRS) in clinical trials with OTC analgesics in headache. Cephalalgia. 2012;32:185-97.

17. Lee HJ, Kim HH, Kim MC, et al. The impact of a high body mass index on laparoscopy assisted gastrectomy for gastric cancer. Surg Endosc. 2009;23:2473-9.

18. Gaszynski T, Szewczyk T. The influence of laparoscopic vs. open gastric bypass on hemodynamic function in morbidly obese patients during general anesthesia. Wideochir Inne Tech Malo Inwazyjne. 2014;9:83-8.

19. Almarakbi WA, Fawzi HM, Alhashemi JA. Effects of four intraoperative ventilatory strategies on respiratory compliance and gas exchange during laparoscopic gastric banding in obese patients. Br J Anaesth. 2009;102:862-8.

20. Huang CM, Lu J. Strategy and prospect of laparoscopic lymph node dissection for locally advanced upper-third gastric cancer. Zhonghua Wei Chang Wai Ke Za Zhi. 2013;16:930-2.

21. Kong J, Wu SD, Su Y. Translumenal single-incision laparoscopy radical gastrectomy with D2 lymph node dissection for early gastric cancer--primary experience with less invasive surgery in China. J Laparoendosc Adv Surg Tech A. 2013;23:141-5.

22. Li Y, Wang J. Station 10 lymph node dissections in laparoscopic-assisted spleen-preserving radical gastrectomy for advanced proximal gastric cancer. Chin J Cancer Res. 2013;25:465-7.

23. Medina-Franco H, Cabrera-Mendoza F, Almaguer-Rosales S, et al. Lymph node ratio as a predictor of survival in gastric carcinoma. Am Surg. 2013;79:284-9.

24. Chen S, Zhao BW, Li YF, et al. The prognostic value of harvested lymph nodes and the metastatic lymph node ratio for gastric cancer patients: results of a study of 1,101 patients. PLoS One. 2012;7:e49424.

25. Lee HJ, Kim HH, Kim MC, et al. The impact of a high body mass index on laparoscopy assisted gastrectomy for gastric cancer. Surg Endosc. 2009;23(11):2473-9.

26. Kim H. Should hand-assisted laparoscopic surgery be placed in the realm of minimally invasive surgery? Ann Coloproctol. 2013;29:42-3. 
27. Patel CB, Ragupathi M, Ramos-Valadez DI, et al. A three-arm (laparoscopic, hand-assisted, and robotic) matched-case analysis of intraoperative and postoperative outcomes in minimally invasive colorectal surgery. Dis Colon Rectum. 2011;54:144-50.

28. Schietroma M, Carlei F, Cecilia EM, et al. A prospective randomized study of systemic inflammation and immune response after laparoscopic nissen fundoplication performed with standard and low-pressure pneumoperitoneum. Surg Laparosc Endosc Percutan Tech. 2013;23:189-96.

29. Pierce A, Pittet JF. Inflammatory response to trauma: implications for coagulation and resuscitation. Curr Opin Anaesthesiol. 2014;27:246-52.

30. Ross C, Byard RW, Langlois NE. Does the intensity of the inflammatory reaction in a bruise depend on its proximity to the site of trauma? Forensic Sci Med Pathol. 2013;9:358-62.

31. Winfield RD, Delano MJ, Dixon DJ, et al. Differences in outcome between obese and nonobese patients following severe blunt trauma are not consistent with an early inflammatory genomic response. Crit Care Med. 2010;38:51-55. 